\title{
The Pattern and Spread of Invasion Can Predict Late Cervical Lymph Node Metastasis in Early Tongue Squamous Cell Carcinoma
}

Koroku Kato ( $\boldsymbol{\nabla}$ koroku@staff.kanazawa-u.ac.jp )

Kanazawa University Graduate School of Medical Science

Hiroki Miyazawa

Kanazawa University Graduate School of Medical Science

Hisano Kobayashi

Kanazawa University Graduate School of Medical Science

Yoshiaki Kishikawa

Kanazawa University Graduate School of Medical Science

Hayato Funaki

Kanazawa University Graduate School of Medical Science

Natsuyo Noguchi

Kanazawa University Graduate School of Medical Science

Kazuhiro Ooi

Kanazawa University Graduate School of Medical Science

Shuichi Kawashiri

Kanazawa University Graduate School of Medical Science

\section{Research Article}

Keywords: Tongue squamous cell carcinoma, Late cervical lymph node metastasis, Mode of invasion, Depth of invasion, Perineural invasion

Posted Date: February 16th, 2022

DOI: https://doi.org/10.21203/rs.3.rs-1349049/v1

License: (c) (i) This work is licensed under a Creative Commons Attribution 4.0 International License.

Read Full License 


\section{Abstract}

To determine the predictive indexes of late cervical lymph node metastasis in early tongue squamous cell carcinoma (TSCC). We retrospectively analyzed the cases of 25 patients with stage I/II TSCC who had undergone surgical treatment without elective neck dissection. We evaluated the relationships between clinicopathologic factors and the occurrence of late cervical lymph node metastasis. Of the 25 cases, metastasis to the cervical lymph nodes was observed in nine cases (36.0\%). The clinicopathological factors associated with late cervical lymph node metastasis were the mode of invasion ( $\mathrm{MOI}, \mathrm{p}=0.032$ ), depth of invasion ( $\mathrm{DOI}, \mathrm{p}=0.004)$, and perineural invasion ( $P N \mathrm{~N}, \mathrm{p}=0.040$ ). A multivariate analysis revealed that only the $\mathrm{DOI}$ was an independent predictor of late cervical lymph node metastasis. The combination of the $\mathrm{DOI}$ and $\mathrm{MOI}$ or the $\mathrm{PNI}$ and $\mathrm{MOI}$ was significantly correlated with late cervical lymph node metastasis ( $p=0.004$ and $p=0.012$, respectively). Our findings suggest that combinations of the MOI, DOI, and PNI could be used as an index for predicting late cervical lymph node metastasis in early TSCC.

\section{Introduction}

Lymph node metastasis is one of the factors in cancer patients that is predictive of prognosis. Tongue squamous cell carcinoma (TSCC) is the most common oral cancer and is characterized by an extensive and well-developed vascular and lymphatic system and a high rate of metastasis to cervical lymph nodes. ${ }^{1}$ Patients with early TSCC generally achieve good outcomes by undergoing the surgical resection of primary sites, ${ }^{2}$ but there some cases of stage I/II TSCC metastasize to one or more cervical lymph nodes after the surgical resection of the primary site and become difficult to control. It was reported that $27 \%-40 \%$ of cervical lymph node metastases in TSCC cases are found at an early stage ${ }^{3}$ and that the probability of late cervical lymph node metastases in early TSCC ranges from $14 \%$ to $29 \% .4,5$

As a method for detecting and evaluating cervical lymph node metastasis, both palpation and imaging examinations are widely used. A clinical examination along with imaging modalities such as magnetic resonance imaging (MRI), computed tomography (CT), ultrasound (US), and positron emission tomography (PET) have been used to detect nodal metastasis. Although the detection sensitivity of lymph node metastasis has been improved by the progress in imaging diagnoses, the detection rate of lymph node metastasis provided by the current imaging test is only $~ 70 \%$, and it not yet possible to detect micrometastases. ${ }^{6,7}$ In order to improve the survival rate of TSCC, it is extremely important to predict and detect the micromeastases to cervical lymph nodes and to deal with them at an early stage.

Many pathological predictors, including immunohistochemical staining, have been studied for cervical lymph node metastasis of TSCC, 8,9 but sensitive and reliable predictors of cervical lymph node metastasis have not yet been identified. We conducted the present study to determine the relationships between the occurrence of late cervical lymph node metastasis and clinicopathological factors in patients with early TSCC, and we examined the effective factors that could be useful to predict late cervical lymph node metastasis in patients with early TSCC. 


\section{Materials And Methods}

\section{Patients and tumor samples}

Of the 29 cases of TSCC at stage I/II that were treated between 2008 and 2017 at Kanazawa University Hospital's Department of Oral and Maxillofacial Surgery, we retrospectively analyzed the cases of the 25 patients who underwent surgery at the primary site as their initial treatment. Prior to the initial surgery, all of the patients were confirmed by contrast-enhanced CT, US, contrast-enhanced MRI and/or FDG-PET to be without cervical lymph node metastasis, distant metastasis, or multiple cancers of other organs. The Union for International Cancer Control (UICC) system (7th and 8th editions) was used for the TNM classification. ${ }^{10,11}$ The nine males and 16 females ranged in age from 30 to 84 years (mean 60.2 yrs, median $65.0 \mathrm{yrs}$ ). All patients were treated with a partial glossectomy without elective neck dissection in the first surgery. The follow-up duration ranged from 20 to 118 months (mean of 57.6 months). Ethical approval for the present study was obtained from the Ethics Committee of the Kanazawa University Graduate School of Medical Science and all methods were performed in accordance with relevant guidelines and regulations. Written informed consent was obtained from each patient.

\section{Clinicopathological parameters}

We examined the relationships between late cervical lymph node metastasis and the following clinicopathological parameters: age, gender, clinical stage ( $7^{\text {th }}$ and $8^{\text {th }}$ UICC editions), histological grade, mode of invasion (MOI) as the index of the pattern of tumor invasion, depth of invasion (DOI), lymphovascular invasion (LVI), and perineural invasion (PNI). The histological grades were classified based on the World Health Organization (WHO) criteria. The MOI values were classified based on the YK classification reported by Yamamoto et al.. ${ }^{12}$ The DOI was measured as the vertical distance from the mucosal surface of the tumor to the deepest point of tissue invasion in millimeters, measured by experienced pathologists.

\section{Statistical analyses}

JMP 13 software program (SAS, Cary, NC, USA) was used to analysis all data. Kaplan-Meier method was used to calculate the 5-year cumulative survival rates and differences were tested by the log-rank test. We used the Fisher's exact test to examine the relationship between late cervical lymph node metastasis and each clinicopathologic parameter. For parameters with significant differences, multivariate analysis was performed using Cox's multivariate proportional hazard model to investigate the parameter's predictive values. The OR value was used to reflect the risk of late cervical lymph node metastasis. A probability value less than 0.05 was judged to be statistically significant.

\section{Results}


In all 25 patients, the excisional margin of the primary site was negative and local recurrence was not observed during the follow-up. Nine of the patients (36\%) had late cervical lymph node metastases, and the average duration from local surgery to the discovery of cervical lymph node metastasis was 5.8 months. A receiver operating characteristics (ROC) curve indicated that the most accurate prediction of late cervical lymph node metastasis was made when the DOI cutoff value was $4 \mathrm{~mm}$. The 5 -year cumulative survival rate for the patients without late cervical lymph node metastasis was understandably $100 \%$, whereas the 5 -year cumulative survival rate for those with late cervical lymph node metastasis was 53.3\% (Fig. 1), confirming that late cervical lymph node metastasis affects the 5-year cumulative survival rate $(\mathrm{p}=0.043)$.

The relationship between clinicopathological factors and late cervical lymph node metastasis

Our analyses of the relationships between clinicopathological factors and late cervical lymph node metastasis revealed, no significant differences between the groups with and without lymph node metastases in age, sex, clinical stage (7th ed.), clinical stage (8th ed.) or LVI, but they did reveal significant between-group differences in the $\mathrm{MOI}, \mathrm{DOI}$ and $\mathrm{PNI}$ and late cervical lymph node metastasis $(p<0.05)$ (Table 1). The results of the multivariate analysis of the three factors with significant differences demonstrated that only the DOI was an independent predictor of late cervical lymph node metastasis (Table 2).

The relationship between combinations of the $\mathrm{MOI}$ and $\mathrm{DO}$ and late cervical lymph node metastasis

We examined whether a combination of $\mathrm{MOI}$, DOI or PNI could predict late lymph node metastasis. We first investigated the relationship between the combination of $\mathrm{MOI}$ and $\mathrm{DOI}$ and late cervical lymph node metastasis (Table 3 ) and observed $55.6 \%$ sensitivity, $93.8 \%$ specificity, the positive predictive value $83.3 \%$ and the negative predictive value $79.0 \%$. In addition, there were significantly fewer cases of late cervical lymph node metastasis among the cases with other than $\mathrm{MOI}$ type $4 \mathrm{D}$ or a $\mathrm{DOI}<4 \mathrm{~mm}(p=0.004)$.

The relationship between combinations of the $\mathrm{MOI}$ and PNI and late cervical lymph node metastasis

We also evaluated the relationship between the combination of the $\mathrm{MOI}$ and PNI and late cervical lymph node metastasis (Table 4), and we observed $55.6 \%$ sensitivity, $93.8 \%$ specificity, the positive predictive value $83.3 \%$, and the negative predictive value $79.0 \%$. There were significantly more cases of late cervical lymph node metastasis among the cases with the combination of MOI type $4 \mathrm{D}$ and PNI-positive status $(p=0.012)$.

\section{Discussion}

The question of whether elective neck dissection should be performed or a conservative observation approach should be taken for cervical lymph nodes in early-stage oral squamous cell carcinoma has been a matter of debate. ${ }^{13,14}$ It was reported that approx. $25 \%$ of patients with TSCC present with occult metastasis at their first medical examination ${ }^{15,16}$ and that not only local recurrence, but also cervical 
lymph node metastases affect overall survival. ${ }^{17-20}$ Our present findings also suggest that late lymph node metastases affect overall survival. There are many reports that elective neck dissection is recommended if the risk of nodal metastasis is $>15 \%,{ }^{21-24}$ but there is another opinion proposing a conservative observation approach in which strict follow-up is performed in order to avoid unnecessary neck dissection. In any case, we believe that the predictive factors for the late cervical lymph nodes metastasis in early TSCC are important and necessary when considering elective neck dissection or as the conditions for strict follow-up by the conservative observation approach.

Many studies have searched for predictors of late cervical lymph node metastasis by using immunohistochemical techniques for primary tumors. The immunohistochemical expression patterns of pan-cytokeratin and podoplanin were reported to be an effective predictor of late cervical lymph node metastasis of TSCC. ${ }^{25}$ A 2004 study attempted to predict late cervical lymph node metastasis by detecting vessels. ${ }^{26}$ Such research requires complicated methods (e.g., immunostaining or specific staining), whereas only the common tissue stain hematoxylin-eosin was the only method needed in the present study. We also used the $\mathrm{MOI}$ as an index; the $\mathrm{MOI}$ was first reported by Yamamoto et al. ${ }^{12}$ and is classified into five types. Type 4D, which has the highest invasive tendency, is highly metastatic. Shimizu et al. reported an association between the $\mathrm{MOI}$ and cervical lymph node metastasis. ${ }^{27}$ In our present analyses as well, there was a significant difference between the $\mathrm{MOI}$ and the occurrence of late cervical lymph node metastasis $(p=0.03)$.

Many data have been published concerning the relationship between the $\mathrm{DO}$ and cervical nodal metastasis with many studies emphasizing its role as a valid predictor. ${ }^{21,24,28-30} 0$-charoenrat et al. used $5 \mathrm{~mm}$ as a cut-off in early oral tongue cancer and demonstrated that the 5 -year survival was $95 \%$ with the tumor thickness of $5 \mathrm{~mm}$ and $30 \%$ when the tumor thickness was $>5 \mathrm{~mm} .{ }^{31} \mathrm{In}$ the current UICC TNM classification (8th ed.), the DOI is incorporated into the T staging, and the DOI has been shown to be an important factor in redefining the staging system, resulting in up-grading based on the DOI cut-off of $5 \mathrm{~mm}$ and $10 \mathrm{~mm} .{ }^{11}$ Our present findings demonstrate that compared to the UICC 7th edition, the clinical staging of the UICC 8th edition shows late cervical lymph nodes metastasis in most cases of stage II, but the difference was not significant.

When we conducted the present investigation with the DOI cut-off set at $4 \mathrm{~mm}$, a significant number of cases of late cervical lymph node metastasis were found among the cases with a DOI $\geq 4 \mathrm{~mm}$. Moreover, our multivariate analysis of the association of the DOI and late cervical lymph node metastasis revealed that the cases with a $\mathrm{DOI} \geq 4 \mathrm{~mm}$ were at a fourfold greater risk of developing late cervical lymph node metastasis compared to cases with a $\mathrm{DOI}<4 \mathrm{~mm}$. Balasubramanian et al. reported that the cervical lymph node metastasis rate was $11.2 \%$ in patients with a $\mathrm{DOI}<4 \mathrm{~mm}$ and $38.5 \%$ in those with a DOI $\geq 4$ $\mathrm{mm}, 32$ and our data support their findings. Moreover, a cut-off point of $4 \mathrm{~mm}$ has traditionally been used to guide the decision for elective neck dissection, based on a study by Kligerman et al.. ${ }^{17}$ These results suggest that even a tumor that has a DOI shallower than the cut-off value of $5 \mathrm{~mm}$ in the UICC $8^{\text {th }}$ edition may cause late cervical lymph node metastasis. 
Other DOIs have been reported to be predictive factors of late cervical lymph node metastasis, ${ }^{33}$ and further validation of the past and present data is required. Herein we observed that when we combined the $\mathrm{DOI}$ and the $\mathrm{MOI}$, in cases with a tumor other than $\mathrm{MOI}$ type $4 \mathrm{D}$ or with a $\mathrm{DOI}<4 \mathrm{~mm}$, the occurrence of late cervical lymph node metastasis was very rare $(p=0.004)$. Moreover, this combination provided $88.9 \%$ sensitivity, $75.0 \%$ specificity, the positive predictive value $66.7 \%$ and the negative predictive value $92.3 \%$. Evaluating the combination of $\mathrm{DOI}$ and $\mathrm{MOI}$ may therefore be useful as a factor for identifying patients who do not develop late lymph node metastasis.

Many of the present patients with PNI had late lymph node metastasis $(p=0.012)$. Lymph node metastasis was observed in another study including patients with $\mathrm{PNI}$, and the patients' prognoses were poor; ${ }^{34}$ we obtained a similar result. We observed that by combining the present of $\mathrm{PNI}$ and the $\mathrm{MOI}$, there were very few cases of late lymph node metastasis among the patients with $\mathrm{MOI}$ type 4D or PNI $(p=0.002)$, and the combination provided $55.6 \%$ sensitivity, $93.8 \%$ specificity, the positive predictive value $83.3 \%$, and the negative predictive value $79.0 \%$. Therefore, evaluating the combination of PNI and the $\mathrm{MOI}$ may be useful as a predictive factor of late cervical lymph node metastasis.

Our study had several limitations. First, this study was based on a small sample size because the incidence of oral cancer is $1.6 \%$ of all malignant neoplasms, ${ }^{35}$ which is not so common, and this study targeted early TSCC without cervical lymph node metastasis at the time of their initial treatment. Second, because our study was a retrospective cohort, there could be missing data. Eventually, despite these limitations, this study provides an interesting relationship between the pattern and spread of invasion in early TSCC and late cervical lymph node metastases.

\section{Conclusion}

By examining the combination of $\mathrm{MOI}$ and $\mathrm{DOI}$ or $\mathrm{PNI}$, we believe that it will be possible to more reliably predict the development of late cervical lymph node metastasis. The present patients who had MOI type $4 \mathrm{D}$, a DOI $\geq 4 \mathrm{~mm}$, or PNI are considered being at high risk of late cervical lymph node metastasis, and elective neck dissection should be considered. If a conservative observation approach is to be taken for such patients, strict follow-up including periodic image examinations is essential, and if cervical lymph node metastasis is suspected, an immediate response should be taken, and it is expected that the prognosis of TSCC can be improved.

\section{Declarations}

\section{Data Availability Statement}

The raw data supporting the conclusions of this article will be made available by the authors.

\section{Ethics approval}


Ethics approval was obtained from Ethics Committee of the Kanazawa University. Written informed consent was obtained from each patient.

\section{Author contributions}

KK conceived the study and wrote the main manuscript text. YK and HF collected data. HM and HK performed statistical analysis. NN and KO prepared table 1-4. SK revised the manuscript. All authors approved the manuscript.

\section{Funding}

Not applicable.

\section{Conflicts of interest}

The authors declare that the research was conducted in the absence of any commercial or financial relationships that could be construed as a potential conflict of interest.

\section{References}

1. Noguti J, De Moura CF, De Jesus GP, Da Silva VH, Hossaka TA, Oshima CT, et al (2012) Metastasis from oral cancer: An overview. Cancer Genomics Proteomics 9:329-335.

2. Zhu L, Wang Y, Li R, Liu A, Zhang X, Zuo C, et al (2019) Surgical treatment of early tongue squamous cell carcinoma and patient survival. Oncol Lett 17:5681-5685. doi: 10.3892/ol.2019.10271.

3. Gosselin BJ. Malignant tumours of the mobile tongue. Medscape. 2015.

4. Kurokawa H, Yamashita Y, Takeda S, Zhang M, Fukuyama H, Takahashi T (2002) Risk factors for late cervical lymph node metastases in patients with stage I or II carcinoma of the tongue. Head Neck 24:731-6. doi: 10.1002/hed.10130.

5. Sparano A, Weinstein G, Chalian A, Yodul M, Weber R (2004) Multivariate predictors of occult neck metastasis in early oral tongue cancer. Otolaryngol Head Neck Surg 131:472-6. doi: 10.1016/j.otohns.2004.04.008.

6. Lim SC, Zhang S, Ishii G, Endoh Y, Kodama K, Miyamoto S, et al (2004) Predictive markers for late cervical metastasis in stage I and II invasive squamous cell carcinoma of the oral tongue. Clin Cancer Res 10:166-172. doi: 10.1158/1078-0432.ccr-0533-3.

7. Khafif RA, Gelbfish GA, Tepper P, Attie JN (1991) Elective radical dissection in epidermoid cancer of the head and neck: A retrospective analysis of 853 cases of mouth, pharynx, and larynx cancer. Cancer 67:67-71. doi: 10.1002/1097-0142(19910101)67:1<67::aid-cncr2820670113>3.0.co;2-a.

8. Chuang ST, Chen CC, Yang SF, Chan LP, Kao YH, Huang MY, et al (2020) Tumor histologic grade as a risk factor for neck recurrence in patients with T1-2NO early tongue cancer. Oral Oncol 106:104706. doi: 10.1016/j.oraloncology.2020.104706. 
9. Almahmoudi R, Kasanen M, Sieviläinen M, Salem A, Pirinen M, Salo T, et al (2019) Prognostic value of blood and lymphatic vessel markers in tongue cancer: A systematic review. Cancer Sci 110:34243433. doi: 10.1111/cas.14189.

10. TNM Classification of Malignant Tumours. In: (2009) International Union against Cancer, LH Sobin, MK Gospodrowicz, CH Wittekind (eds.), 7th Ed. Wiley-Liss, New York, NY.

11. TNM Classification of Malignant Tumours. In: (2016) International Union against Cancer, JB Brierley, MK Gospodrowicz, CH Wittekind (eds.), 8th Ed. Wiley-Liss, New York, NY.

12. Yamamoto E, Kohama G, Sunagawa H, Iwai M, Hiratsuka H (1983) Mode of invasion, bleomycin sensitivity, and clinical course in squamous cell carcinoma of the oral cavity. Cancer 51:2175-2180. doi: 10.1002/1097-0142(19830615)51:12<2175::aid-cncr2820511205>3.0.co;2-m.

13. Oh LJ, Phan K, Kim SW, Low TH, Gupta R, Clark JR (2020) Elective neck dissection versus observation for early-stage oral squamous cell carcinoma: Systematic review and meta-analysis. Oral Oncol 105:104661. doi: 10.1016/j.oraloncology.2020.104661.

14. Ibrahim SA, Ahmed ANA, Elsersy HA, Darahem IMH (2020) Elective neck dissection in T1/T2 oral squamous cell carcinoma with NO neck: Essential or not? A systematic review and meta-analysis. Eur Arch Otorhinolaryngol 277:1741-1752. doi: 10.1007/s00405-020-05866-3.

15. Ahmed F, Islam KM (1990) Site of predilection of oral cancer and its correlation with chewing and smoking habits. a study of 103 cases. Bangladesh Med Res Counc Bull;16 17-25.

16. Faisal M, Abu Bakar M, Sarwar A, Adeel M, Batool F, Malik Kl, et al (2018) Depth of invasion (DOI) as a predictor of cervical nodal metastasis and local recurrence in early stage squamous cell carcinoma of oral tongue (ESSCOT). PLoS One 13:e0202632. doi: 10.1371/journal.pone.0202632.

17. Kligerman J, Lima RA, Soares JR, Prado L, Dias FL, Freitas EQ, et al (1994) Supraomohyoid neck dissection in the treatment of T1/T2 squamous cell carcinoma of oral cavity. Am J Surg 168:391394. doi: 10.1016/s0002-9610(05)80082-0.

18. Moratin J, Metzger K, Engel M, Hoffmann J, Freudlsperger C, Freier K, et al (2019) The occurrence of cervical metastases in squamous cell carcinoma of the tongue: Is there a rationale for bilateral neck dissection in early stage tumors? J Craniomaxillofac Surg 47:1134-1138. doi: 10.1016/j.jcms.2019.03.003.

19. Akhtar S, Ikram M, Ghaffar S (2007) Neck involvement in early carcinoma of tongue. Is elective neck dissection warranted? J Pak Med Assoc 57:305-307.

20. D'Cruz AK, Vaish R, Kapre N, Dandekar M, Gupta S, Hawaldar R, et al (2015) Elective versus therapeutic neck dissection in node-negative oral cancer. N Engl J Med 373:521-529. doi: 10.1056/NEJMoa1506007.

21. Kowalski LP, Medina JE (1998) Nodal metastases: Predictive factors. Otolaryngol Clin North Am 31:621-637. doi: 10.1016/s0030-6665(05)70076-1.

22. Huang SH, Hwang D, Lockwood G, Goldstein DP, O'Sullivan B (2009) Predictive value of tumor thickness for cervical lymph-node involvement in squamous cell carcinoma of the oral cavity: A meta-analysis of reported studies. Cancer 115:1489-1497. doi: 10.1002/cncr.24161. 
23. Keski-Säntti H, Atula T, Törnwall J, Koivunen P, Mäkitie A (2006) Elective neck treatment versus observation in patients with T1/ T2 N0 squamous cell carcinoma of oral tongue. Oral Oncol 42:96101. doi: 10.1016/j.oraloncology.2005.06.018.

24. Melchers LJ, Schuuring E, van Dijk BA, de Bock GH, Witjes MJ, van der Laan BF, et al (2012) Tumour infiltration depth $\geq 4 \mathrm{~mm}$ is an indication for an elective neck dissection in $\mathrm{pT} 1 \mathrm{cN} 0$ oral squamous cell carcinoma. Oral Oncol 48:337-342. doi: 10.1016/j.oraloncology.2011.11.007.

25. Hmada M, Ebihara Y, Nagata K, Yano M, Kogashiwa Y, Nakahira M, et al (2020) Podoplanin is an efficient predictor of neck lymph node metastasis in tongue squamous cell carcinoma with low tumor budding grade. Oncol Lett 19:2602-2608. doi: 10.3892/ol.2020.11358.

26. Tanigaki Y, Nagashima Y, Kitamura Y, Matsuda H, Mikami Y, Tsukuda M (2004) The expression of vascular endothelial growth factor- $A$ and $-C$, and receptors 1 and 3: Correlation with lymph node metastasis and prognosis in tongue squamous cell carcinoma. Int $\mathrm{J}$ Mol Med 14:389-395.

27. Shimizu S, Miyazaki A, Sonoda T, Koike K, Ogi K, Kobayashi Jl, et al (2018) Tumor budding is an independent prognostic marker in early stage oral squamous cell carcinoma: With special reference to the mode of invasion and worst pattern of invasion. PLoS One 13:e0195451. doi: 10.1371/journal.pone.0195451.

28. Gourin CG, Conger BT, Porubsky ES, Sheils WC, Bilodeau PA, Coleman TA (2008) The effect of occult nodal metastases on survival and regional control in patients with head and neck squamous cell carcinoma. Laryngoscope 118:1191-1194. doi: 10.1097/MLG.0b013e31816e2eb7.

29. Frech S, Hörmann K, Riedel F, Götte K (2009) Lymphatic vessel density in correlation to lymph node metastasis in head and neck squamous cell carcinoma. Anticancer Res 29:1675-1679.

30. Foschini MP, Leonardi E, Eusebi LH, Farnedi A, Poli T, Tarsitano A, et al (2013) Podoplanin and Ecadherin expression in preoperative incisional biopsies of oral squamous cell carcinoma is related to lymph node metastases. Int J Surg Pathol 21:133-141. doi: 10.1177/1066896912471851.

31. O-charoenrat P, Pillai G, Patel S, Fisher C, Archer D, Eccles S, et al (2003) Tumour thickness predicts cervical nodal metastases and survival in early oral tongue cancer. Oral Oncol 39:386-390. doi: 10.1016/s1368-8375(02)00142-2.

32. Balasubramanian D, Ebrahimi A, Gupta R, Gao K, Elliott M, Palme CE, et al (2014) Tumor thickness as a predictor of nodal metastases in oral cancer: Comparison between tongue and floor of the mouth subsites. Oral Oncol 50:1165-1168. doi: 10.1016/j.oraloncology.2014.09.012.

33. Tam S, Amit M, Zafereo M, Bell D, Weber RS (2019) Depth of invasion as a predictor of nodal disease and survival in patients with oral tongue squamous cell carcinoma. Head Neck 41:177-184. doi: 10.1002/hed.25506.

34. Haug K, Breuninger H, Metzler G, Eigentler T, Eichner M, Häfner HM, et al (2020) Prognostic impact of perineural invasion in cutaneous squamous cell carcinoma: results of a prospective study of 1,399 tumors. J Invest Dermatol 140:1968-1975. doi: 10.1016/j.jid.2020.01.035.

35. Global Burden of Disease Cancer Collaboration, Fitzmaurice C, Abate D, Abbasi N, Abbastabar H, Abd-Allah F, et al (2019) Global, Regional, and National Cancer Incidence, Mortality, Years of Life 
Lost, Years Lived With Disability, and Disability-Adjusted Life-Years for 29 Cancer Groups, 1990 to 2017.: A systematic analysis for the global burden of disease study. JAMA Oncol. 5:1749-1768. doi: 10.1001/jamaoncol.2019.2996.

\section{Tables}

Table 1. Clinicopathological parameters in relation to late cervical lymph node metastasis in patients with TSCC. 


\begin{tabular}{|c|c|c|c|c|}
\hline \multirow[b]{2}{*}{ Parameters } & \multirow[b]{2}{*}{$\mathbf{n}$} & \multicolumn{3}{|c|}{ Late cervical lymph node metastasis } \\
\hline & & + & - & $p$ value \\
\hline \multicolumn{2}{|l|}{ Age, yrs: } & & & 0.79 \\
\hline$<65$ & 12 & 4 & 8 & \\
\hline$\geq 65$ & 13 & 5 & 8 & \\
\hline \multicolumn{2}{|l|}{ Sex: } & & & 0.282 \\
\hline Male & 9 & 2 & 7 & \\
\hline Female & 16 & 7 & 9 & \\
\hline \multicolumn{3}{|c|}{ Clinical stage (UICC 7th Ed.): } & & 0.271 \\
\hline T1N0 & 12 & 3 & 9 & \\
\hline T2NO & 13 & 6 & 7 & \\
\hline \multicolumn{3}{|c|}{ Clinical stage (UICC 8th Ed.): } & & 0.093 \\
\hline T1N0 & 8 & 1 & 7 & \\
\hline T2NO & 17 & 8 & 9 & \\
\hline \multicolumn{2}{|c|}{ Histological grade: } & & & 0.188 \\
\hline Grade 1 & 8 & 1 & 7 & \\
\hline Grade 2 & 10 & 4 & 6 & \\
\hline Grade 3 & 7 & 4 & 3 & \\
\hline MOI: & & & & 0.033 \\
\hline Type 1 & 2 & 0 & 2 & \\
\hline Type 2 & 5 & 1 & 4 & \\
\hline Type 3 & 11 & 4 & 7 & \\
\hline Type 4C & 3 & 0 & 3 & \\
\hline Type 4D & 4 & 4 & 0 & \\
\hline DOI: & & & & 0.011 \\
\hline$<4 \mathrm{~mm}$ & 14 & 1 & 12 & \\
\hline$\geq 4 \mathrm{~mm}$ & 11 & 8 & 4 & \\
\hline
\end{tabular}




\begin{tabular}{|lcccc|}
\hline LVl: & & & & 0.053 \\
\hline- & 12 & 2 & 10 & \\
\hline & 13 & 7 & 6 & \\
\hline PNI: & & & & 0.022 \\
\hline- & 20 & 5 & 15 & \\
\hline & 5 & 4 & 1 & \\
\hline
\end{tabular}

Table 2. Multivariate analyses of clinicopathological parameters in relation to late cervical lymph node metastasis in patients with TSCC.

\begin{tabular}{|lllll|}
\hline & & P-value & & \\
\hline Factor & Groups & Univariate & Multivariate & OR $(95 \% \mathrm{Cl})$ \\
\hline MOI & Other than type 4D/type 4D & 0.032 & 0.1368 & \\
\hline DOI & $<4 \mathrm{~mm} / \geq 4 \mathrm{~mm}$ & 0.004 & 0.0286 & $12.299(0.977-154.657)$ \\
\hline PNI & $-/+$ & 0.040 & 0.4378 & \\
\hline
\end{tabular}

Table 3. Relationship between the combination of $\mathrm{MOI}$ and $\mathrm{DOI}$ and late cervical lymph node metastasis

\begin{tabular}{|llll|}
\hline & \multicolumn{2}{c|}{$\begin{array}{c}\text { Late cervical lymph } \\
\text { node metastasis }\end{array}$} \\
\hline MOI type 4D or DOI $\geq 4 \mathrm{~mm}$ & + & - & $\mathbf{n}$ \\
\hline MOI other than type 4D or DOI <4mm & 1 & 12 & 13 \\
\hline $\mathbf{n}$ & 9 & 4 & 12 \\
\hline
\end{tabular}

Table 4. Relationship between the combination of $\mathrm{MOI}$ and PNI and late cervical lymph node metastasis 


\begin{tabular}{|lccc|}
\hline & \multicolumn{3}{c|}{$\begin{array}{c}\text { Late cervical lymph } \\
\text { node metastasis }\end{array}$} \\
\hline MOI type 4D or PNI positive & $\square$ & - & $\mathbf{n}$ \\
\hline MOI other than type 4D or PNI negative & 4 & 15 & 19 \\
\hline $\mathbf{n}$ & 5 & 1 & 6 \\
\hline
\end{tabular}

Figures

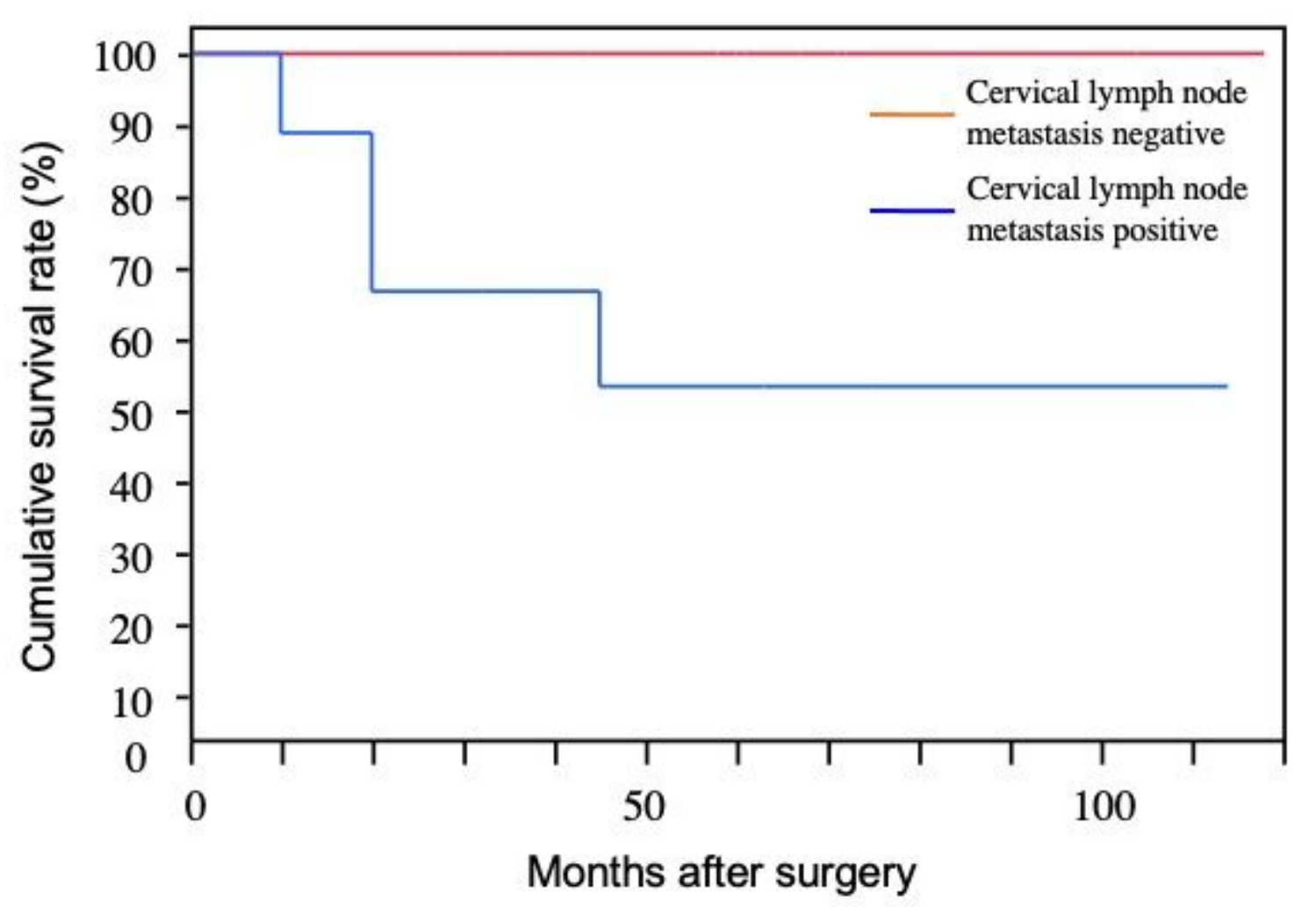

Fig. 1

Figure 1

Kaplan-Meier estimates for 5-year cumulative survival based on late cervical lymph node metastasis. The 5-year cumulative survival rate of patients with late cervical lymph node metastasis (53.3\%) was 
significantly lower than that of patients without it $(100 \%)(p=0.0043)$. 